\title{
Antithrombotic therapy in pediatric ventricular assist devices: Multicenter survey of the European EXCOR Pediatric Investigator Group
}

The International Journal of Artificial Organs

2018, Vol. 41 (7) 385-392

(C) The Author(s) 2018

Reprints and permissions: sagepub.co.uk/journalsPermissions.nav DOI: 10.1 177/0391398818773040 journals.sagepub.com/home/jao @SAGE

\author{
Oliver Miera', Katharina L Schmitt', Hakan Akintuerk'2, \\ Angele Boet ${ }^{3}$, Robert Cesnjevar ${ }^{4}$, Teresa Chila ${ }^{5}$, Thilo Fleck ${ }^{6}$, \\ Ranny Goldwasser ${ }^{7}$, Luis G Guereta ${ }^{8}$, Beatrice Heineking', \\ Juergen Hoerer ${ }^{3}$, Alexander Horke' ${ }^{10}$, Tain Y Hsia'", \\ Michael Huebler'2, Andrzej Kansy'3, Ann Karimova'", \\ Bohdan Maruszewski ${ }^{13}$, Constancio Medrano ${ }^{14}$, Szymon Pawlak ${ }^{15}$, \\ Zdenka Reinhardt ${ }^{5}$, Birgitta Romlin ${ }^{16}$, Eugen Sandica ${ }^{17}$, \\ Florian Schmidt ${ }^{10}$, René Schramm 9 , Martin Schweiger ${ }^{12}$, \\ Joanna Śliwka ${ }^{15}$, Brigitte Stiller ${ }^{6}$, Josef Thul ${ }^{2}$ and Antonio Amodeo ${ }^{18}$
}

\footnotetext{
Abstract

Objectives: Mechanical circulatory support for pediatric heart failure patients with the Berlin Heart EXCOR ventricular assist system is the only approved and established bridging strategy for recovery or heart transplantation. In recent years, the burden of thromboembolic events has led to modifications of the recommended antithrombotic therapy. Therefore, we aimed to assess modifications of antithrombotic practice among the European EXCOR Pediatric Investigator Group members.

Methods: We sent a questionnaire assessing seven aspects of antithrombotic therapy to 18 European hospitals using the EXCOR device for children. Returned questionnaires were analyzed and identified antithrombotic strategies were

'Department of Congenital Heart Disease-Pediatric Cardiology, Deutsches Herzzentrum Berlin, Berlin, Germany

2Pediatric Heart Center, University Hospital Giessen, Justus Liebig University, Giessen, Germany

${ }^{3}$ Cardiac ICU, "Marie Lannelongue" Surgical Center, South Paris University Hospitals, Paris, France

${ }^{4}$ Department of Pediatric Cardiac Surgery, University Hospital Erlangen, Erlangen, Germany

${ }^{5}$ Department of Pediatric Cardiology, Freeman Hospital, Newcastle Upon Tyne, UK

${ }^{6}$ Department of Congenital Heart Disease and Pediatric Cardiology, University Heart Center Freiburg, Freiburg, Germany

${ }^{7}$ Department of Pediatric Cardiology/Congenital Heart Disease, Heidelberg University Hospital, Heidelberg, Germany

${ }^{8}$ Pediatric Cardiology, University Hospital La Paz, Madrid, Spain

${ }^{9}$ Department of Pediatric Cardiology and Pediatric Intensive Care, Ludwig-Maximilians-University Munich, Munich, Germany

${ }^{10}$ Department of Pediatric Cardiology and Intensive Care Medicine, Medical School Hannover, Hannover, Germany

"Department of Pediatric Cardiology, Great Ormond Street Hospital for Children, London, UK

${ }^{12}$ Department of Congenital Pediatric Surgery, Zurich Children's Hospital, Zurich, Switzerland

${ }^{13}$ Department of Cardiothoracic Surgery, The Children's Memorial Health Institute, Warsaw, Poland

${ }_{14}$ Pediatric Cardiology Division, Hospital Materno Infantil, Hospital General Universitario Gregorio Marañón, Madrid, Spain

${ }^{15}$ Department of Cardiac Surgery and Transplantology, Silesian Center for Heart Diseases, Zabrze, Poland

${ }^{16}$ Department of Pediatric Anesthesia and Intensive Care, Queen Silvia Children's Hospital, Gothenburg, Sweden

${ }^{17}$ Center for Congenital Heart Defects, Heart and Diabetes Center NRW, Bad Oeynhausen, Germany

${ }^{18}$ Department of Pediatric Cardiology and Cardiac Surgery, Bambino Gesù Children's Hospital, Rome, Italy
}

Corresponding author:

Oliver Miera, Department of Congenital Heart Disease-Pediatric Cardiology, Deutsches Herzzentrum Berlin, Augustenburger Platz I, Berlin 13353, Germany.

Email: miera@dhzb.de 
descriptively compared to "Edmonton protocol" recommendations developed for the US EXCOR pediatric approval study.

Results: Analysis of 18 received surveys revealed substantial deviations from the Edmonton protocol, including earlier start of heparin therapy at 6-12h postoperatively and in $50 \%$ of surveyed centers, monitoring of heparin effectiveness with aPTT assay, administering vitamin $\mathrm{K}$ antagonists before 12 months of age. About $39 \%$ of centers use higher international normalized ratio targets, and platelet inhibition is changed in $56 \%$ including the use of clopidogrel instead of dipyridamole. Significant inter-center variability with multiple deviations from the Edmonton protocol was discovered with only one center following the Edmonton protocol completely.

Conclusion: Current antithrombotic practice among European EXCOR users representing the treatment of more than 600 pediatric patients has changed over time with a trend toward a more aggressive therapy. There is a need for systematic evidence-based evaluation and harmonization of developmentally adjusted antithrombotic management practices in prospective studies toward revised recommendations.

\section{Keywords}

Anticoagulation, Berlin Heart, EXCOR pediatric, platelet inhibition, ventricular assist device

Date received: 28 February 2018; accepted: 5 March 2018

\section{Introduction}

The EXCOR ventricular assist device (EXCOR) is clinically used since 1990 for circulatory support of pediatric heart failure in almost 2000 patients as a bridge to transplant and bridge to recovery strategy. ${ }^{1,2}$ The blood-contacting surfaces of EXCOR pumps are covalently coated with heparin (CARMEDA CBAS; Carmeda, Upplands Väsby, Sweden) to enhance hemocompatibility. However, similar to other ventricular assist device (VAD) antithrombotic therapy (AT) is necessary to reduce the risk of thromboembolic complications inherently induced by VAD-blood interactions. ${ }^{3-5}$ Since the hemostatic system in children undergoes significant postnatal development, ${ }^{6-8}$ a developmentally adjusted AT in the postoperative care of pediatric patients is required.

In the early years, EXCOR patients were kept on intravenous unfractionated heparin (UFH) to achieve activating clotting times of $140-160 \mathrm{~s}$. Vitamin $\mathrm{K}$ antagonists (VKA) and platelet inhibitors (PI) were not used. Antithrombin III was substituted if it fell below 70\%. ${ }^{9}$ This approach, however, led to frequent bleeding complications. Around the year 2000, the activated partial thromboplastin time (aPTT) was introduced for UFH therapeutic monitoring. Furthermore, older children now received VKA with a target international normalized ratio (INR) level of 2.7-3.5, and PI therapy (dipyridamole and acetylsalicylic acid (ASA)) was added after the first postoperative week. Efficacy of AT treatment was monitored by thromboelastography (TEG; Haemonetics Corporation, Braintree, MA, USA) and platelet function tests with target activation of $30 \%$. These adaptations resulted in decreased pump exchange rates and mortality. ${ }^{10}$

A systematic pediatric EXCOR AT guideline ("Edmonton protocol (EP)") was introduced for the first time during the US EXCOR pediatric pivotal approval study, incorporating recommendations from the hematology literature and clinical experience from the cumulative global pediatric EXCOR use. ${ }^{11,12}$ The EP further became the recommended AT strategy for pediatric patients by Berlin Heart.
Despite the EP, the incidence of thromboembolic neurologic complications among US EXCOR pediatric centers varied between 0 and 1.7 per 100 patient days. ${ }^{13}$ This observation motivated some centers to modify the EP based on their own cumulative clinical experience. Hence, today it is not known to what extent EXCOR users adhere to the EP. Therefore, a standardized, paper-based survey was developed by and distributed within the European EXCOR Pediatric Investigator Group (EEPIG). The aim of this survey was to generate qualitative and quantitative data on the current AT practice.

\section{Materials and methods}

A standardized non-validated paper-based survey was developed with the consensus of the EEPIG by the investigator team to cover the following aspects: availability of AT protocol, use of UFH, antithrombin III, low-molecular-weight heparin (LMWH), VKA, PI, and institutional AT management practice (see Supplementary material). The survey was constructed using closed questions, multiple-choice elements, numerical data, and free text elements and distributed to the EEPIG in October 2015. Ethical Committee approval was waived because no patient-related data were collected. Simple measures of distribution were used for descriptive statistical analysis. Results were compared to the EP recommendations as published in detail elsewhere. ${ }^{11,12}$ The core recommendations of the EP are as follows. (1) UFH is initiated $24-48 \mathrm{~h}$ postimplantation when bleeding is minimal. The main modality for monitoring of UFH is the anti-factor Xa level, with a target range of $0.35-0.5 \mathrm{U} / \mathrm{mL}$ and corresponding aPTT 1.52.5 times baseline. (2) Switching from UFH to LMWH is done $48 \mathrm{~h}$ postoperatively (target anti-FXa range: $0.6-1.0 \mathrm{U} /$ $\mathrm{mL}$ ). (3) In children over 1 year of age, anticoagulation should be switched to oral VKA (target INR 2.7-3.5). (4) Dipyridamole is recommended as the first and ASA as the second PIs. Dipyridamole (started $48 \mathrm{~h}$ postimplantation at $4 \mathrm{mg} / \mathrm{kg} /$ day) and ASA (started after chest tube removal at $1 \mathrm{mg} / \mathrm{kg} /$ day) must be dosed individually based on the results of TEG Platelet Mapping assays. 
Table I. Unfractionated heparin therapy.

\begin{tabular}{|c|c|c|c|c|c|c|c|c|}
\hline \multirow[t]{2}{*}{ Site } & \multicolumn{2}{|c|}{ Age independent } & \multicolumn{3}{|c|}{ Age $<12$ months } & \multicolumn{3}{|c|}{ Age $>12$ months } \\
\hline & $\begin{array}{l}\text { Start time } \\
\text { (hours post- } \\
\text { op) }\end{array}$ & Platelets $(\mu \mathrm{L})$ & $\begin{array}{l}\text { Initial dose } \\
\text { (IU/kg/h) }\end{array}$ & aPTT target (s) & $\begin{array}{l}\text { Anti-FXa } \\
\text { target }(\mathrm{IU} / \mathrm{mL})\end{array}$ & $\begin{array}{l}\text { Initial dose } \\
(\mathrm{IU} / \mathrm{kg} / \mathrm{h})\end{array}$ & aPTT target (s) & $\begin{array}{l}\text { Anti-FXa } \\
\text { target }(\mathrm{IU} / \mathrm{mL})\end{array}$ \\
\hline EP & $24-28$ & $>20,000$ & 15 & & $0.35-0.5$ & 10 & & $0.35-0.5$ \\
\hline 1 & 24 & $>50,000$ & 10 & $50-80$ & - & - & $50-80$ & - \\
\hline 2 & 24 & - & 15 & - & $0.6-1.0$ & 10 & - & $0.6-1.0$ \\
\hline 3 & 24 & - & 15 & - & $0.35-0.5$ & 10 & - & $0.35-0.5$ \\
\hline 4 & $24-48$ & $>80,000$ & 10 & $60-80$ & - & 8 & $50-70$ & - \\
\hline 5 & $12-24$ & $>50,000$ & 4 & $40-50$ & - & 4 & $40-50$ & - \\
\hline 6 & $20-24$ & $>100,000$ & 10 & $40-60$ & - & 10 & $40-50$ & - \\
\hline 7 & 12 & $>50,000$ & 16 & - & $0.35-0.7$ & 16 & - & $0.35-0.7$ \\
\hline 8 & 24 & $>50,000$ & 25 & - & $0.37-0.7$ & 25 & - & $0.37-0.7$ \\
\hline 9 & 24 & $>100,000$ & 25 & $60-80$ & $0.5-1.0$ & 15 & $60-80$ & $0.5-1.0$ \\
\hline 10 & $12-18$ & $>100,000$ & 13 & $60-80$ & - & 13 & $60-80$ & - \\
\hline 11 & $6-8$ & $>50,000$ & 8 & $70-90$ & - & 8 & $60-80$ & - \\
\hline 12 & 8 & - & 8 & $60-80$ & $0.7-0.9$ & 8 & $60-80$ & $0.7-0.9$ \\
\hline 13 & 24 & - & 15 & $>72$ & - & 10 & $>72$ & - \\
\hline 14 & 12 & - & NA & - & - & 10 & $60-70$ & - \\
\hline 15 & $24-48$ & $>20,000$ & 15 & $2 \times-3 \times$ baseline & $0.6-1.0$ & 10 & $2 \times-3 \times$ baseline & $0.6-1.0$ \\
\hline 16 & $18-24$ & $>20,000$ & 15 & $60-80$ & $0.35-0.7$ & 10 & $60-80$ & $0.35-0.7$ \\
\hline 17 & 24 & $>50,000$ & 15 & $\begin{array}{l}1.5 \times-2.5 \times \\
\text { baseline }\end{array}$ & $0.35-0.5$ & 10 & $\begin{array}{l}1.5 \times-2.5 \times \\
\text { baseline }\end{array}$ & $0.35-0.5$ \\
\hline 18 & $8-12$ & $>30,000$ & $4-8$ & $60-80$ & - & $4-8$ & $60-80$ & - \\
\hline
\end{tabular}

aPTT: activated partial thromboplastin time; anti-FXa: anti-factor Xa; EP: Edmonton protocol. Recommendations of the EP are given in the first line.

\section{Results}

A total of 18/18 hospitals from France, Germany, Italy, Poland, Spain, Sweden, Switzerland, and United Kingdom returned the surveys for analysis. These hospitals represent a cumulative clinical experience of 621 pediatric EXCOR patients supported between 1990 and 2016.

\section{Availability of an antithrombotic protocol}

About $10(56 \%)$ of the hospitals perform AT based on a modified EP, whereas only $5(29 \%)$ follow the original recommendations. Three centers (17\%) do not use written procedures for EXCOR pediatric AT management.

\section{UFH}

Of the 18 surveyed hospitals, nine (50\%) hospitals reported earlier initiation of UFH between 6 and $20 \mathrm{~h}$ postoperatively (Table 1). However, starting dose of UFH is lower for infants in $8(44 \%)$ hospitals and for children in $5(28 \%)$. About 10 $(56 \%)$ of the hospitals are requiring a higher platelet count above $50,000 / \mu \mathrm{L}$ as a minimum. Thromboelastography criteria for the initiation of UFH therapy are rarely used.

A majority of 12 hospitals $(67 \%)$ reported using only one type of assay. About 13 (72\%) centers use aPTT, while
$9(50 \%)$ use the recommended anti-FXa assay. Three hospitals (17\%) use activated clotting time for UFH therapy monitoring. Only $5(28 \%)$ hospitals use aPTT and antiFXa assays together in line with the EP recommendation. Target ranges for aPTT are shown in Table 1.

\section{Antithrombin III substitution}

Antithrombin III monitoring and substitution therapy during UFH application as bolus $(67 \%)$, infusion $(17 \%)$ or using fresh frozen plasma (11\%) were reported by 17 (94\%) of the hospitals. The lower threshold triggering antithrombin III substitution is $80 \%$ in eight $(44 \%)$ hospitals; in two (11\%) centers, a target of $100 \%$ is used. Eight (44\%) centers use a threshold consistent with the EP of $70 \%$.

\section{LMWH}

About $12(67 \%)$ centers use LMWH therapy and $6(33 \%)$ only in the youngest patients that are unable to take oral anticoagulants (Table 2). Only five (28\%) of the hospitals fully switch to LMWH therapy before starting VKA. If used, LMWH is initiated 1-14 days postoperatively. Two centers (11\%) use UFH for long-term anticoagulation in children under 6 months of age. 
Table 2. Long-term anticoagulation.

\begin{tabular}{|c|c|c|c|c|c|}
\hline Site & Medication & Age range (months) & Start at post-op day & $\begin{array}{l}\text { Anti-FXa target (U/ } \\
\mathrm{mL})\end{array}$ & $\begin{array}{l}\text { Age at initiation of } \\
\text { VKA (months) }\end{array}$ \\
\hline EP & Enoxaparin & $0-12$ & 2 & $0.6-1$ & 12 \\
\hline 1 & Enoxaparin & $0-8$ & $2-3$ & $0.6-1$ & 8 \\
\hline 2 & Enoxaparin & $0-12$ & 3 & $0.7-1$ & 12 \\
\hline 3 & Enoxaparin & $0-12$ & 3 & $0.6-1$ & 12 \\
\hline 4 & Enoxaparin & $0-12$ & $2-3$ & $0.7-1$ & 12 \\
\hline 5 & Enoxaparin & $0-6$ & $5-7$ & $0.6-1$ & 6 \\
\hline 6 & $N A^{a}$ & & & & 0 \\
\hline 7 & $N A^{a}$ & & & & 0 \\
\hline 8 & $N A^{a}$ & & & & 3 \\
\hline 9 & $N A^{a}$ & & & & 0 \\
\hline 10 & UFH & $0-6$ & I & & 6 \\
\hline 11 & Nadroparin & $0-12$ & $3-5$ & $0.8-1.2$ & 12 \\
\hline 12 & Enoxaparin & $0-24$ & $3-4$ & $0.7-0.9$ & 24 \\
\hline 13 & Enoxaparin & $0-12$ & 3 & $0.6-1$ & 12 \\
\hline 14 & $N A^{a}$ & & & & 0 \\
\hline 15 & Enoxaparin & $0-12$ & I & $0.6-1$ & 12 \\
\hline 16 & Enoxaparin & $0-24$ & $7-14$ & $0.7-1.2$ & 12 \\
\hline 17 & Enoxaparin & $0-12$ & $b$ & $0.5-0.6$ & 12 \\
\hline 18 & UFH & $0-6$ & I & & 6 \\
\hline
\end{tabular}

Anti-FXa: anti-factor Xa; VKA: vitamin K antagonist; UFH: unfractionated heparin; EP: Edmonton protocol.

Drug, age range, starting time, anti-FXa targets, and age at initiation of vitamin $\mathrm{K}$ antagonist.

asite does not use heparin for long-term anticoagulation.

bTime to initiation varies substantially depending on condition of patient, need for drainages, and so on.

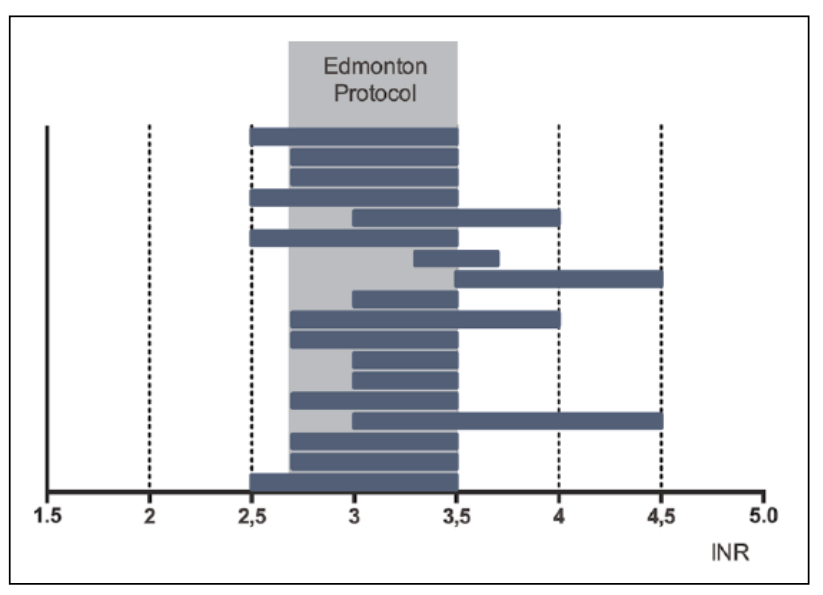

Figure I. INR target ranges. Each bar represents individual center with recommendations according to the Edmonton protocol shown in gray.

\section{VKAs}

Of all surveyed hospitals, nine $(50 \%)$ initiate VKA therapy at $<12$ months of age (Table 2). Warfarin is used as VKA in 13 $(72 \%)$ of the centers, phenprocoumon in $4(22 \%)$, and acenocoumarol in $1(6 \%)$. VKA therapy is started in $4(22 \%)$ of the hospitals within the first week postoperative, the remaining $14(78 \%)$ hospitals initiate VKA therapy solely based on the individual clinical status of the patient. The majority of hospitals followed the EP INR target range. However, a substantial proportion of centers aimed for higher upper INR targets of up to 4.5 (five centers, 28\%) and higher lower limits of INR 3-3.5 (seven centers, 39\%; Figure 1).

\section{Platelet inhibition}

A huge variety of PI therapy algorithms were identified by the survey. Criteria reported by the hospitals for PI therapy initiation are tolerability of enteral feeding, minimum platelet count, cessation of all bleeding, stop of draining from drainages, as well as results of platelet monitoring. Of all surveyed centers, $13(72 \%)$ use minimum platelet count as a requirement for the initiation of PI therapy, whereas other criteria have reported considerably less frequently. The minimum platelet count in all but one hospital using this criterion must be higher than recommended by the EP, eight (44\%) of all centers use a minimum platelet count of $80,000-100,000 / \mu \mathrm{L}$.

About 12 (67\%) of the evaluated hospitals changed the platelet inhibition from EP, with $61 \%$ prescribing ASA as the first drug and 50\% using clopidogrel as the second drug (Table 3).

ASA daily doses, when given as first PI, vary between 0.5 and $30 \mathrm{mg} / \mathrm{kg}$ with a frequency of $1-4$ doses per day. Clopidogrel as second PI is given at daily doses of $0.25-$ $0.6 \mathrm{mg} / \mathrm{kg}$ with a frequency of $1-4$ doses per day. 
Table 3. Platelet inhibition therapy.

\begin{tabular}{llll}
\hline Combination first PI/second PI & $\mathrm{n}(\%)$ & Initiation first PI (POD, range) & Initiation second PI (POD, range) \\
\hline ASA/clopidogrel $^{\mathrm{a}}$ & $8(44)$ & $2-5$ & $3-8$ \\
Dipyridamole/ASA $^{\mathrm{b}}$ & $6(33)$ & $2-4$ & $3-5$ \\
ASA/dipyridamole & $2(1 \mathrm{I})$ & $3-10$ & $5-14$ \\
Dipyridamole/ASA/clopidogrel & $\mathrm{I}(6)$ & $2-4$ & $4-7$ \\
Only ASA & $\mathrm{I}(6)$ & 5 & - \\
\hline
\end{tabular}

PI: platelet aggregation inhibitor; POD: postoperative day; ASA: acetylsalicylic acid.

Drugs used for platelet inhibition and time of initiation.

a One site initiates antiplatelet therapy with dipyridamole followed by ASA and switches from dipyridamole to clopidogrel in the second week of treatment.

bSequence dipyridamole/ASA is recommended in the Edmonton protocol.

For routine monitoring of PI therapy a variety of assays, activators and thresholds are being used. Five (28\%) of the hospitals routinely use the TEG platelet mapping assay (TEG; Haemonetics Corporation), eight (44\%) impedance aggregometry (Multiplate; Roche Diagnostics International, Rotkreuz, Switzerland, or ROTEM; Tem International, Basel, Switzerland), and light transmission aggregometry (BioChemica, Flacht, Germany) in one. The remaining four centers $(22 \%)$ did not routinely use any assay for platelet monitoring. If clinically indicated, for example, bleeding or deposit formation within the pump chamber different tests is applied in these centers (TEG platelet mapping, light transmission, impedance aggregometry each, and a combination of all tests in one center).

\section{Institutional antithrombotic management practice}

The AT of pediatric EXCOR patients in most centers is managed by intensivists (55\%), cardiologists (44\%), the VAD team $(17 \%)$, and surgeons (22\%). Only four hospitals (22\%) involve hematologists in the management of AT therapy.

\section{Other modifications of the EP}

Three hospitals (17\%) reported to routinely use methylprednisolone for the management of inflammatory episodes, hyperfibrinogenemia, or sepsis, and one hospital reported to substitute factor XIII in case of deficiency of this factor.

\section{Assessment of deviation from $E P$}

The most clinically relevant deviations from the EP identified in this survey are as follows:

1. UFH earlier initiated than in EP, UFH efficacy monitoring mostly based on aPTT, often no age adjustment of UFH dosages in infants;

2. LMWH other than enoxaparin is used;
3. VKA is started in patients aged below 12 months;

4. Lower and upper INR efficacy targets are increased;

5. PI therapy is mostly implemented by sequential ASA/clopidogrel;

6. Therapeutic monitoring of platelet inhibition is performed with other methods than TEG platelet monitoring or preserved for special circumstances.

\section{Discussion}

Compared to adults pediatric patients require adaption of AT therapy due to significant developmental differences of the concentration and activity of multiple elements of the hemostatic system and drug metabolism. ${ }^{14-22}$ These developmental differences imply that AT regimen developed for adult patients cannot be simply transferred to newborns, infants, children, and adolescents. Despite the introduction of the EP in 2007, 10\%-29\% of pediatric EXCOR patients experience at least one ischemic neurologic complication. ${ }^{23-27}$

The analysis of risk factors for ischemic neurologic complications identified in pediatric EXCOR patient cohorts supports a significant impact of age and morbidity on hemostasis and AT needs. Among the risk factors identified in smaller studies are younger age, lower body weight, congenital heart disease (CHD) etiology and pump size to body surface area ratio. ${ }^{28-30}$ However, in the largest multicenter prospective cohort of 204 children, risk stratification for neurological events was not possible based on preimplantation characteristics. ${ }^{23}$ Clinical experience shows that the risk of cerebral bleeding is lower compared to the risk for ischemic cerebrovascular events in pediatric EXCOR patients. With regard to clinical evidence, the predominance of ischemic neurologic complications observed in the large US post-approval multicenter study also among the US EXCOR centers has raised the question whether AT recommended in the EP needs to be changed. ${ }^{26}$

In their evaluation of the use of EP in the EXCOR VAD Investigational Device Exemption Study, Steiner et al. ${ }^{31}$ 
Table 4. Deviations in local antithrombotic protocols from Edmonton protocol.

\begin{tabular}{|c|c|c|c|c|}
\hline Number of centers & 18 & 5 & 8 & 5 \\
\hline Cases per center & & $1-10$ & $11-30$ & $>30$ \\
\hline Cumulative cases & 621 & 26 & 150 & 445 \\
\hline Time to UFH initiation earlier & $9(50)$ & $3(60)$ & $4(50)$ & $2(40)$ \\
\hline Monitoring of UFH with aPTT & II (6I) & $4(80)$ & $4(50)$ & $3(60)$ \\
\hline LMWH not used long-term & $6(33)$ & $2(40)$ & I (13) & $3(60)$ \\
\hline First PI other than dipyridamole & $10(56)$ & $4(80)$ & $3(38)$ & $3(60)$ \\
\hline Second PI clopidogrel & $9(50)$ & $3(60)$ & $5(63)$ & I (20) \\
\hline Monitoring other than TEG PM & $13(72)$ & $5(100)$ & $6(75)$ & $2(40)$ \\
\hline Age at initiation of VKA & $9(50)$ & $4(80)$ & $2(25)$ & $3(60)$ \\
\hline Lower INR target increased & $7(39)$ & $2(40)$ & $3(38)$ & $2(40)$ \\
\hline Upper INR target increased & $5(28)$ & $2(40)$ & $I(13)$ & $2(40)$ \\
\hline
\end{tabular}

UFH: unfractionated heparin; aPTT: activated partial thromboplastin time; LMWH: low-molecular-weight heparin; PI: platelet aggregation inhibitor; TEG PM: thromboelastography platelet mapping; VKA: vitamin $\mathrm{K}$ antagonist; INR: international normalized ratio.

Data expressed as number (percentage). Results are shown in regard to center experience (total case load low (I-I0)—-medium (I I-30)—high (>30)).

observed that only one-third of neurological events were attributed to AT intensity and concluded that the incidence of stroke may be decreased by revisions to the existing protocol. Among considerations to improving the existing AT protocol were earlier initiation of ASA and earlier therapeutic anti-Xa levels, as well as different methods of monitoring antiplatelet effects. ${ }^{31}$ Recently, Rosenthal et al. ${ }^{32}$ published promising results with a more aggressive platelet inhibition in 11 children on VAD, including triple antiplatelet therapy, uptitration to high dosages, and ASA as the first drug to be introduced. Interestingly, many of the surveyed centers had already implemented many of these modifications to their existing protocols.

Our survey is the first systematic multi-institutional evaluation of current antithrombotic real-life practice among EXCOR users in pediatric patients. It shows that relevant modifications of the EP have evolved, and that there is significant heterogeneity between AT protocols among hospitals. It could be shown that a majority of EEPIG members initiate UFH therapy early, but at recommended dosing, and use aPTT instead of anti-FXa for therapeutic UFH monitoring. Main variations in VKA therapy are use in very young patients, even newborns, and higher INR targets. PI therapy has been changed toward a sequence of ASA and clopidogrel with targets of $>70 \%$ inhibition of induced aggregation and the introduction of new tests. It appears that many hospitals follow more aggressive AT protocols intended to lower the incidence of adverse thromboembolic events. Center experience does not influence the amount of changes made in the AT protocol (Table 4).

Due to the low incidence of terminal heart failure in children, the large heterogeneity of patient cohorts and the variation in AT practice, there is little robust evidence as to the effectiveness of AT strategy variations on thromboembolic complication rates. Moreover, little is known how often and how centers deviate from standardized protocols due to individual clinical patient conditions like bleeding, infection, observance of thrombotic deposits within the pump chamber, congenital abnormalities, or hemostatic disorders. To overcome this shortcoming, the EEPIG members proposed a harmonized modified AT protocol which might be used in future prospective studies on AT in pediatric EXCOR patients (see Supplementary material).

The heterogeneity of AT approaches detected in this survey implies that AT in children on EXCOR support is challenging and that contemporary pediatric AT practice might not sufficiently address the complexities of this patient population.

Our study has several limitations related to the study design. The questionnaire was not validated and reported protocols do not reflect deviations in clinical practice. More importantly, outcome data are missing. Therefore, no conclusion in regard to efficacy or safety of any of the protocols and modifications was drawn. The decision to not collect data on adverse events was made for several reasons. Centers may have changed their AT protocol with time, adverse event data were not collected prospectively making them prone to underreporting, and standard deviation of event rates in an individual center was high due to the low number of cases, precluding comparability between centers. Reporting event rates in such circumstances would be misleading and might induce erroneous conclusions leading to the preference of a seemingly superior AT protocol.

\section{Conclusion}

This survey reveals that current AT practice among Berlin Heart EXCOR users for pediatric patients has changed over time in comparison with the EP and that it varies substantially among centers. Given the higher risk for ischemic cerebrovascular events compared to the risk of cerebral bleeding, the majority of centers implemented more aggressive antithrombotic protocols. Apparently, there is a need not only for more contemporary, evidence-based AT 
recommendations but also for more systematic clinical research and finally evidence-based AT guidelines for this group of patients. The data from this analysis can serve as a starting point toward developing a revised, more aggressive EXCOR pediatric AT guideline which shall be validated in a prospective multicenter cohort study.

\section{Acknowledgements}

The authors thank Giang Tong for editorial assistance.

\section{Declaration of conflicting interests}

The author(s) declared the following potential conflicts of interest with respect to the research, authorship, and/or publication of this article: O.M., R.G., and A.K. received travel fees from Berlin Heart. K.L.S. has received consultant/travel fees from Berlin Heart. The remaining authors have no conflicts of interest to disclose.

\section{Funding}

The author(s) disclosed receipt of the following financial support for the research, authorship, and/or publication of this article: This work was supported by the Stiftung Charité (BIH_PRO_430 to O.M.).

\section{References}

1. Hetzer R, Kaufmann F and Delmo Walter EM. Paediatric mechanical circulatory support with Berlin Heart EXCOR: development and outcome of a 23-year experience. Eur $J$ Cardiothorac Surg 2016; 50(2): 203-210.

2. Stiller B, Benk C and Schlensak C. Congenital heart disease: mechanical cardiovascular support in infants and children. Heart 2011; 97(7): 596-602.

3. Fraser KH, Zhang T, Taskin ME, et al. A quantitative comparison of mechanical blood damage parameters in rotary ventricular assist devices: shear stress, exposure time and hemolysis index. J Biomech Eng 2012; 134(8): 081002.

4. Yu X, Larsen B, Rutledge J, et al. The profile of the systemic inflammatory response in children undergoing ventricular assist device support. Interact Cardiovasc Thorac Surg 2012; 15(3): 426-431.

5. Stiller B, Lemmer J, Merkle F, et al. Consumption of blood products during mechanical circulatory support in children: comparison between ECMO and a pulsatile ventricular assist device. Intensive Care Med 2004; 30(9): 1814-1820.

6. Awad M, Czer LS, Soliman C, et al. Prevalence of warfarin genotype polymorphisms in patients with mechanical circulatory support. ASAIO J 2015; 61(4): 391-396.

7. Baghai M, Heilmann C, Beyersdorf F, et al. Platelet dysfunction and acquired von Willebrand syndrome in patients with left ventricular assist devices. Eur J Cardiothorac Surg 2015; 48(3): 421-427.

8. Baldwin ACW, Sandoval E, Letsou GV, et al. Surgical approach to continuous-flow left ventricular assist device explantation: a comparison of outcomes. J Thorac Cardiovasc Surg 2016; 151: 192-198.

9. Hetzer R, Loebe M, Potapov EV, et al. Circulatory support with pneumatic paracorporeal ventricular assist device in infants and children. Ann Thorac Surg 1998; 66(5): 1498-1506.
10. Hetzer R, Potapov EV, Stiller B, et al. Improvement in survival after mechanical circulatory support with pneumatic pulsatile ventricular assist devices in pediatric patients. Ann Thorac Surg 2006; 82(3): 917-925.

11. Fraser CD Jr, Jaquiss RDB, Rosenthal DN, et al. Prospective trial of a pediatric ventricular assist device. $N$ Engl $J$ Med 2012; 9367(6): 532-541.

12. Almond CS, Buchholz H, Massicotte P, et al. Berlin Heart EXCOR pediatric ventricular assist device investigational device exemption study: study design and rationale. $\mathrm{Am}$ Heart J 2011; 162(3): 425-426.

13. Almond CS, Lorts A, Rossano JW, et al. US center variability in the incidence of stroke for the Berlin Heart EXCOR pediatric ventricular assist device. Circulation 2015; 6: A19540.

14. Guzzetta NA, Miller BE, Todd K, et al. Clinical measures of heparin's effect and thrombin inhibitor levels in pediatric patients with congenital heart disease. Anesth Analg 2006; 103(5): 1131-1138.

15. Guzzetta NA and Miller BE. Principles of hemostasis in children: models and maturation. Paediatr Anaesth 2011; 21(1): 3-9.

16. Andrew M, Paes B and Johnston M. Development of the hemostatic system in the neonate and young infant. Am J Pediatr Hematol Oncol 1990; 12(1): 95-104.

17. Andrew M, Paes B, Milner R, et al. Development of the human coagulation system in the healthy premature infant. Blood 1988; 72(5): 1651-1657.

18. Andrew M, Paes B, Milner R, et al. Development of the human coagulation system in the full-term infant. Blood 1987; 70(1): 165-172.

19. Kuhle S, Male C and Mitchell L. Developmental hemostasis: pro- and anticoagulant systems during childhood. Semin Thromb Hemost 2003; 29(4): 329-338.

20. Shah JK, Mitchell LG, Paes B, et al. Thrombin inhibition is impaired in plasma of sick neonates. Pediatr Res 1992; 31(4 Pt 1): 391-395.

21. Corrigan JJ, Sleeth JJ, Jeter M, et al. Newborn's fibrinolytic mechanism: components and plasmin generation. $\mathrm{Am} \mathrm{J}$ Hematol 1989; 32(4): 273-278.

22. Parmar N, Albisetti M, Berry LR, et al. The fibrinolytic system in newborns and children. Clin Lab 2006; 52(3-4): 115-124.

23. Jordan LC, Ichord RN, Reinhartz O, et al. Neurological complications and outcomes in the Berlin Heart EXCOR $\AA$ pediatric investigational device exemption trial. J Am Heart Assoc 2015; 4(1): e001429-100149.

24. Cassidy J, Dominguez T, Haynes S, et al. A longer waiting game: bridging children to heart transplant with the Berlin Heart EXCOR device - the United Kingdom experience. $J$ Heart Lung Transplant 2013; 32(11): 1101-1106.

25. Anyanwu AC, Itagaki S, Pinney S, et al. Initial experience with routine less invasive implantation of HeartMate II left ventricular assist device without median sternotomy. Eur $J$ Cardiothorac Surg 2014; 46(6): 985-990.

26. Almond CS, Morales DL, Blackstone EH, et al. Berlin Heart EXCOR pediatric ventricular assist device for bridge to heart transplantation in US children. Circulation 2013; 127(16): 1702-1711.

27. Sandica E, Knyphausen EZ, Blanz U, et al. Safety of longterm mechanical support with Berlin heart EXCOR in pediatric patients. World J Pediatr Congenit Heart Surg 2012; 13(1): 72-76. 
28. Miera O, Schmitt KRL, Delmo-Walter E, et al. Pump size of Berlin heart EXCOR pediatric device influences clinical outcome in children. J Heart Lung Transplant 2014; 33(8): 816-821.

29. Polito A, Netto R, Soldati M, et al. Neurological complications during pulsatile ventricular assistance with the Berlin Heart EXCOR in children: incidence and risk factors. Artif Organs 2013; 37(10): 851-856.

30. Byrnes JW, Prodhan P, Williams BA, et al. Incremental reduction in the incidence of stroke in children supported with the Berlin EXCOR ventricular assist device. Ann Thorac Surg 2013; 96(5): 1727-1733.

31. Steiner ME, Bomgaars LR, Massicotte MP, et al. Antithrombotic therapy in a prospective trial of a pediatric ventricular assist device. ASAIO J 2016; 62(6): 719727.

32. Rosenthal DN, Lancaster CA, McElhinney DB, et al. Impact of a modified anti-thrombotic guideline on stroke in children supported with a pediatric ventricular assist device. J Heart Lung Transplant 2017; 36: 1250-1257. 